the type of service covering their home district unless their paediatrician had a diabetes liaison service which covered them.

Time off school, diabetes related problems in school, school visits by the liaison service, teachers' knowledge of diabetes, and parental satisfaction with support and information in school were compared between children covered by paediatric and by adult services.

\section{Results}

Adult services covered $226(65 \%)$ children, paediatric services $103(29 \%)$, and no service was identified for $22(6 \%)$. There was no difference in mean age of children covered by adult and by paediatric services.

No significant differences were found between children with adult and paediatric services in the time lost from school, or in the proportions with diabetes related problems in school.

For only 171 (49\%) children had there been a school visit by the liaison service. Children covered by paediatric services were 1.5 times ( $95 \%$ confidence interval $1 \cdot 2$ to $1 \cdot 7, p=0.001$ ) more likely to get a school visit with $67(65 \%)$ doing so compared with $104(46 \%)$ of those covered by adult services. Parents whose children received a paediatric service were also more satisfied with information and support in school (table). Overall, 200 (57\%) parents said teachers lacked know-ledge of diabetes, but this proportion did not differ between adult and paediatric services.

When parents were asked to comment about diabetes services, 292 (55\%) of 531 comments concerned school. Of these, $198(67 \%)$ related to teachers' lack of knowledge, 103 of which mentioned management of diet and of hypoglycaemia. Thirty comments concerned poor communication between teachers or uncaring attitudes, although 14 said the school was caring and understanding. Other comments concerned difficulties with school trips, mood changes, and deterioration in school work. Parents made 37 comments on improving school care, 31 suggesting school visits by professionals, and six suggesting an educational video for teachers.
Parental satisfaction with school support provided by adult and paediatric diabetes liaison services; number (\%)

\begin{tabular}{lcc}
\hline Satisfaction & Adult & Paediatric \\
\hline Very satisfied & $24(13)$ & $26(29)$ \\
Satisfied on the whole & $88(46)$ & $43(47)$ \\
Somewhat dissatisfied & $42(22)$ & $15(17)$ \\
Very dissatisfied & $36(19)$ & $7(7)$ \\
Total $^{\star}$ & $190(100)$ & $91(100)$
\end{tabular}

«48 'don't know'.

$\chi^{2}$ for trend $=13 \cdot 5, \mathrm{df}=1, \mathrm{p}=0.0002$.

\section{Discussion}

Children with diabetes spend considerable time at school; this time should be both tolerable and safe. The BDA recommends a school visit for every child, followed up at least yearly. ${ }^{6}$ Disappointingly it appears that many paediatric nurses did not visit schools, although they were more likely to than adult nurses. Parents were more satisfied with paediatric nurses, but this did not improve their opinions of teachers' knowledge.

We therefore recommend that a trained paediatric diabetes nurse should talk to the class teacher, games teacher, dinner lady, playground supervisors, school doctor and nurse, before the child returns to school. Information must cover the main areas of concern, hypoglycaemia and diet. Information packs and telephone numbers should be supplied. The paediatric diabetes nurse should revisit the school at least yearly and liaise with the school health service.

MAT holds an MRC Health Services Research Training Fellowship. We thank Adele McEvilly for her help.

1 Bradbury AJ, Smith CS. An assessment of the diabetic knowledge of school teachers. Arch Dis Child 1983; 58: 692-6.

2 Warne J. Diabetes in school: a study of teachers' knowledge and information sources. Practical Diabetes 1988; 5: $210-5$.

3 Challen AH, Davies AG, Williams RJW, Baum JD. Support for families with diabetic children: parents' views. Practical Diabetes 1990; 7: 26-31.

4 Kauffman L, Bradbury AJ, Comer L. Experience of diabetic children and their families in relation to diet at school.
chen $\mathrm{L}$, Bradbury AJ, Comer L. Experience of diabetic Practical Diabetes 1990; 4: 166-70.

5 Lessing DN, Swift PGF, Baum JD, Metcalfe MA. Newly diagnosed diabetes: a study of parental satisfaction. Arch Dis Child 1992; 67: 1011-3.

6 British Diabetic Association Committee. What professional supervision should children with diabetes and their families expect? London: British Diabetic Association, 1989.
Hospital for Sick

Children, London, Department of Infectious Diseases

Julian Shield

Vas Novelli

Diana Gibb

Department of

Gastroenterology

Colin Melville

Peter Milla

Department of

Histopathology

Glenn Anderson

Irene Scheimberg

Correspondence and reprint requests to: Dr Vas Novelli, Infectious Diseases Unit,

Hospital for Sick Children,

Great Ormond Street,

Great Ormond Street,
London WC1N 3JH.

Accepted 6 June 1993

\section{Bovine colostrum immunoglobulin concentrate for cryptosporidiosis in AIDS}

\author{
Julian Shield, Colin Melville, Vas Novelli, Glenn Anderson, Irene Scheimberg, Diana \\ Gibb, Peter Milla
}

\author{
Abstract \\ Lactobin- $R$ is a commercial hyperimmune \\ bovine colostrum with potent anticrypto- \\ sporidial activity. It was administered to a \\ 4 year old child with AIDS and severe diar- \\ rhoea associated with cryptosporidiosis.
}

There was significant clinical improvement in the diarrhoea and permanent elimination of the parasite from the gut as assessed through serial jejunal biopsy and stool specimens.

(Arch Dis Child 1993; 69: 451-453) 
Although the parasite cryptosporidium causes a self limiting diarrhoea illness in immunocompetent patients, it may be associated with life threatening diarrhoea in those with immunodeficiency. ${ }^{1}$ Uniformly effective treatment is unavailable at present, and immunotherapies, such as hyperimmune bovine colostrum, have given mixed results. ${ }^{2-4}$ We describe a 4 year old boy with AIDS whose cryptosporidiosis was successfully treated with a commercial hyperimmune bovine colostrum preparation.

\section{Case report}

A 4 year old boy with vertically acquired HIV infection had a one month history of severe watery diarrhoea, vomiting, and failure to thrive. He was malnourished (weight of $13 \mathrm{~kg}$, $<3$ rd centile) with generalised lymphadenopathy and hepatosplenomegaly. Stool examination revealed cryptosporidia but no other pathogens. He was severely immunodeficient with a CD4 count of $0.19 \times 10^{9} / 1$, but was negative for $\mathrm{p} 24$ antigen. He had specific iron, copper, and zinc deficiencies, and was hypoalbuminaemic (albumin concentration $30 \mathrm{~g} / \mathrm{l}$ ).

Two weeks of oral spiramycin, at a dose of $100 \mathrm{mg} / \mathrm{kg} / \mathrm{day}$, had no effect on the diarrhoea or weight loss. Cryptosporidial oocysts continued to be isolated from the stool. Total parenteral nutrition was instituted, but regrading to enteral feeds was impossible due to vomiting. Azithromycin was given intravenously for three weeks at a dose of $2.5 \mathrm{mg} / \mathrm{kg}$ twice daily, and oral loperamide and subcutaneous diamorphine were added. Although there was a clear reduction in stool volume, he remained intolerant of feeds, and oocysts persisted in his stools.

Lactobin-R (Biotest) was administered for two weeks in a dose of $50 \mathrm{~g} /$ day, made up to $500 \mathrm{ml}$ in sterile water, and given via nasogastric tube and feeding pump over 12 hours. A specimen from a jejunal biopsy performed immediately before starting this treatment showed severe crypt hyperplastic partial villous atrophy and cryptosporidia, confirmed on electron microscopy, adherent not only to the epithelial surface but also to the crypts (figure A). No acid-alcohol fast bacilli were seen. Stool output did not decrease significantly during treatment. The patient, however, tolerated both the bovine colostrum and enteral feeds. Serial stool samples sent, after the commencement of Lactobin-R treatment, were negative for cryptosporidia. A repeat jejunal biopsy specimen after two weeks of treatment showed a marked improvement in villous architecture and absence of cryptosporidia (figure B). Six months after completion of treatment, stool samples had remained negative for cryptosporidia and were of normal volume and consistency. Unfortunately, the patient died of an encephalopathic illness soon after. No cryptosporidia were seen on postmortem examination.

\section{Discussion}

The increased severity of cryptosporidiosis in patients with immunodeficiency and the lack of effective chemotherapeutic agents have encouraged attempts at immunological intervention. In the mouse model, it has been shown that the active anticryptosporidial component of bovine colostrum lies in the immunoglobulin fraction, ${ }^{5}$ which presumably acts by interfering with parasite adherence to the gastrointestinal epithelium, although the exact mechanism is unknown.

We treated our patient with a new preparation consisting of an immunoglobulin concentrate, prepared from bovine colostrum (Lactobin-R). Despite the continuing severity of his immunodeficiency, the child's diarrhoea stopped and he was able to tolerate enteral feeds. His stools became permanently oocystfree, and specimens from jejunal biopsies performed before and after treatment showed elimination of the infection and marked improvement in mucosal architecture.

Lactobin- $R$ is the immunoglobulin fraction purified from the first colostrum of veterinary attested cattle. They are screened for foot and mouth disease, bovine viral diarrhoea, rotavirus, and mastitis. During production the colostrum is heated to $70^{\circ} \mathrm{C}$ and the $\mathrm{pH}$ reduced to 4.5 to inactivate herpes and sendai viruses. It is then lyophilised into $50 \mathrm{~g}$ sachets. Contamination with micro-organisms is at low levels. The preparation contains $87 \%$ $\mathrm{IgG}_{1}$, and small amounts of $\operatorname{IgA}$ and IgM. Anticryptosporidial titres of $50 \mathrm{~g} / 500 \mathrm{ml}$ solutions range from $1: 2560$ to $1: 5120$ in enzyme linked immunosorbent assay (ELISA) assays using cleaned cryptosporidial oocysts as the antigen. The same solution has significant titres against Escherichia coli, klebsiella,
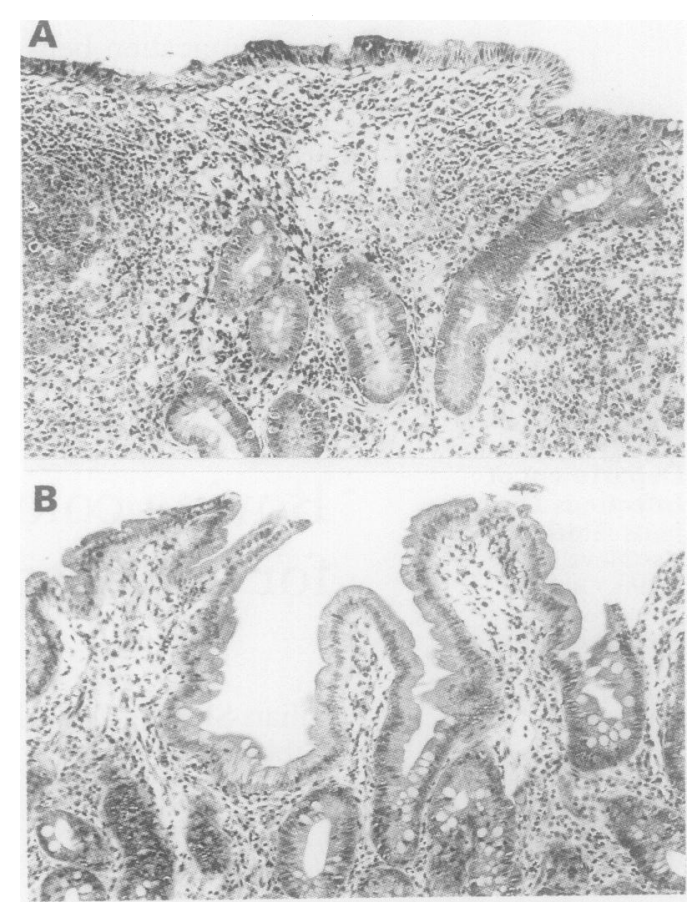

Fejunal biopsy specimens before and after treatment (haemotoxylin and eosin, magnification $\times 40$ ).

(A) Specimen shows crypt hyperplasia, partial villous atrophy, and cryptosporidia adherent to crypts and surface epithelium. (B) Specimen shows villous architecture much improved and no cryptosporidia seen. 
pseudomonas, Staphylococcus aureus, and Staphylococcus epidermidis (J Nrunjun, personal communication).

In immunocompetent patients cryptosporidia only infect villous enterocytes and parasites are not found low down on the villus or in the crypts. ${ }^{1}$ In our patient with compromised mucosal immunity there were cryptosporidia clearly adherent to crypt epithelium in a manner reminiscent of a patient previously reported with IgA deficiency. ${ }^{6} \mathrm{We}$ suggest that infestation of the crypts is related to lack of mucosal immunoglobulin; leads to reduced parasite elimination by secondary mucosal defences of mucus secretion and peristalsis; and may accentuate fluid loss by targeting crypt secretion either directly or via cryptosporidial toxins (Quarino, unpublished observations, 1992).

Oral hyperimmune bovine colostrum, obtained from cows immunised specifically with cryptosporidial antigens, has previously been used to treat cryptosporidiosis in immunodeficient patients. It transiently cleared parasites from one child with congenital hypogammaglobulinaemia and chronic cryptosporidiosis, but after three weeks there was biliary tract relapse. ${ }^{2}$ In a pilot study of the use of hyperimmune bovine colostrum (immunoflorescence titres of 1:3200-1:25 600) in five patients with AIDS, one patient had marked reduction in oocyst excretion, one a moderate reduction, and the third no reduction, while neither of the control patients showed any change. ${ }^{3}$ Another group reported a further case of cryptosporidiosis in an AIDS patient responding to treatment with hyperimmune bovine colostrum. The illness recurred after three months but this may have been due to reinfection rather than relapse. ${ }^{4}$

This is the first reported case of a commercially available bovine immunoglobulin concentrate used successfully to treat chronic cryptosporidiosis in an immunodeficient individual resulting in permanent clearance of stools and mucosa. Further studies of Lactobin- $R$, including efficacy trials in immunodeficient populations such as those with AIDS, seem warranted.

We would like to thank Professor W P Duffus, department of veterinary medicine, University of Bristol, for helpful advice, and BIOTEST Pharma GmbH, Dreieich, Germany, for supplying the concentrate. 1 Current WL, Garcia LS. Cryptosporidiosis. Clin Microbiol
Rev 1991; 4: 325-58.

2 Tzipori S, Roberton D, Chapman C. Remission of diarrhoea due to cryptosporidiosis in an immunodeficient child treated with hyperimmune bovine colostrum. BMF 1986;

3 Nord J, Marp J, Dijohn D, Tzipori S, Tacket CO. Treatment with bovine hyper-immune colostrum of cryptosporidial with bovine hyper-immune colostrum of cryptosp.
diarrhoea in AIDS patients. AIDS 1990; 14: 581-4.

4 Unger BLP, Ward DJ, Fayer R, Quinn CA. Cessation of cryptosporidium associated diarrhoea in an acquired immunodeficiency syndrome patient after treatment with hyper-immune colostrum. Gastroenterology 1990; 98: 486-9.

5 Fayer R, Guidry A, Blayburn BL. Immunotherapeutic efficacy of bovine colostral immunoglobulins from a hyperimmunised cow against cryptosporidiosis in neonatal mice. Infect Immun 1990; 58: 2962-5.

6 Jacyna MR, Parkin J, Goldin R, Baron JH. Protracted enteric cryptosporidial infection in selective immunoglobulin $A$ and saccharomyces opsonin deficiencies. Gut 1990; 31: 714-6.

\title{
Occurrence of Duchenne dystrophy in Klinefelter's syndrome
}

\author{
V Ramesh, R Mountford, H M Kingston, A Kelsey, M J Noronha, M A Clarke
}

Department of

Neurology, Royal

Manchester Children's

Hospital

V Ramesh

A Kelsey

M J Noronha

M A Clarke

Regional Molecular

Genetics Laboratory,

St Mary's Hospital,

Manchester

R Mountford

H M Kingston

Correspondence to: Dr V Ramesh, Department of Neurology, Royal Manchester Children's Hospital, Pendlebury,
Manchester M27 1HA

Accepted 29 June 1993

\begin{abstract}
A boy with Duchenne muscular dystrophy and facial dysmorphism in conjunction with Klinefelter's genotype $47 \mathrm{XXY}$ is presented; this is an unusual situation with two genetic errors evolving over two generations. Karyotyping should be considered in boys with Duchenne muscular dystrophy who have unusual features. (Arch Dis Child 1993; 69: 453-454)
\end{abstract}

A case of Klinefelter's syndrome and Becker muscular dystrophy was reported in $1989 .{ }^{1}$ We report a boy with Klinefelter's syndrome and Duchenne muscular dystrophy where two genetic errors were responsible. An Xp21 nondeletion dystrophin gene mutation in the mother was passed on in a double dose to the child because of maternal meiotic nondysjunction; this has not been described before.

\section{Case report}

A 3.5 year old boy presented with symptoms of proximal muscle weakness, speech delay, and a serum creatine kinase activity of $24750 \mathrm{U} / \mathrm{l}$. Because of mild facial dysmorphism with hypertelorism and a prominent nose, a cytogenetic analysis was performed and revealed a 47XXY karyotype. There was no history of neuromuscular disease in the family, but his 57 year old maternal grandfather had been in a wheelchair from early adult life for apparently untreated Perthe's disease.

Examination revealed a boy with mild facial dysmorphism including hypertelorism and a prominent nose with height and weight on the 3rd and head circumference below the 3rd centile. He had bulky calves with pelvic girdle weakness. A percutaneous needle biopsy specimen from the quadriceps muscle showed a dystrophic process. On immunohistochemistry only a few fibres were positive for dystrophin 1 and 2. Psychometric assessment at 6 